\title{
Differences in primary indication for guide-extension catheter usage among the three target vessels
}

\author{
Ruka Yoshida $^{1}$, Hideki Ishii ${ }^{1}$, Akihito Tanaka ${ }^{1}$, Iwana Inukai ${ }^{2}$, Kensuke Takagi ${ }^{3}$, Toyoaki Murohara ${ }^{1}$ \\ ${ }^{1}$ Department of Cardiology, School of Medicine, Nagoya University Graduate, Nagoya, Japan \\ ${ }^{2}$ Science Education Association, Japan \\ ${ }^{3}$ Department of Cardiology, Ogaki Municipal Hospital, Ogaki, Japan
}

Adv Interv Cardiol 2020; 16, 2 (60): 192-197

DOI: https://doi.org/10.5114/aic.2020.96063

\section{Introduction \\ During complex percutaneous coronary intervention $(\mathrm{PCI})$, one of the biggest challenges is the inability to cross devices to the target lesions [1]. The buddy wire and anchoring balloon technique can be helpful in such situations [2, 3]. The mother-in-child technique is anoth- er powerful method $[4,5]$. Applying a similar concept, the rapid-exchange guide extension catheter (GEC) has been evolving. Previous studies have proposed a mechanism of its efficacy as increasing back-up support by deep in- tubation, as a conduit to decrease friction between the vessel wall and the catheter, or for improving co-axial alignment between the catheter and the lesion $[6,7]$. However, little is known regarding the differences in pri- mary indication with respect to the target vessels.}

\section{Aim}

The purpose of this study was to clarify the primary indication for GEC according to the target vessels and to provide thorough consideration of the mechanistic effect of GEC.

\section{Material and methods}

We enrolled 232 consecutive patients with 251 lesions (excluding left main and bypass-graft lesions) for whom GEC-facilitated PCI was performed between March 2014 and September 2018. Those who participated in another study were excluded [8]. The research review board of our hospital approved this study, which complied with the Helsinki Declaration. All patients provided consent for participation in this study.

GuideLiner V3 (Vascular Solutions Inc., Minneapolis, MN, USA), Guidezilla (Boston Scientific, Marlborough,
Massachusetts, USA), or GuidePlus (Nipro Corp., Osaka, Japan) was used. The primary indication for GEC was defined as follows [6, 7]: 1) to increase back-up support; 2) as an outer sheath; 3) to facilitate co-axial alignment between the catheter and the lesion; 4) to selectively inject contrast; and 5) other. Although the complete differentiation among the indication of 1)-3), especially between 1) and 2), was hard to achieve, we defined the indication of 1)-3) as follows: the indication of 1) was defined in cases where the operators simply would like to increase backup support of a guiding catheter to facilitate delivering stents or a balloon with little awareness of the tortuosity or angle of the vessel proximal to the target lesion; indication of 2) was defined in cases where the GECs have passed beyond the lesions and the devices were delivered; indication of 3) was defined in cases where the tortuosity or acute bending proximal to the target lesions might underlie the difficulty to deliver devices (especially, short monorail with floppy shaft, such as intravascular ultrasound). Two experienced operators (R.Y. and K.T.) independently reviewed angiography and decided the primary indication. Any discrepancies between the two operators were resolved by consensus after consulting a third operator (Y.M.). Procedural success was defined as $<20 \%$ residual stenosis with thrombolysis in myocardial infarction 3 flow in the target vessel, and device success was defined as delivering GEC to the desired position. Tortuosity was defined as proximal segment angulation of $>90^{\circ}$ and calcification was visually assessed [7].

\section{Results}

The mean age of the patients was $73.3 \pm 10.2$ years and three-quarters of patients were men. Approximately

\section{Corresponding author:}

Ruka Yoshida MD, Department of Cardiology, School of Medicine, Nagoya University Graduate, Nagoya, Japan, phone: +81527412111, e-mail: lyoshida@hotmail.com

Received: 17.01.2020, accepted: 17.03.2020. 
one-half of the patients had diabetes mellitus, chronic kidney disease, or prior history of $\mathrm{PCl}$. Angiographic and procedural characteristics are presented in the Table I. Calcified lesions were more frequent in the right coronary artery (RCA) and left anterior descending (LAD) artery than in the left circumflex artery (LCX), which resulted in more frequent use of rotational atherectomy in the RCA and LAD. On the other hand, proximal tortuosity was more frequent in the LCX.

The primary indication for GEC is shown in Figure $1 \mathrm{~A}$. The primary indication was similar for the RCA and LAD: increasing back-up of the guiding catheter was the most common reason, whereas improving alignment was the most common reason for the LCX. The indication for selective contrast injection was less common in all the three vessels. The device success rate was numerically lower in the LCX compared with the RCA and LAD. Therefore, the incidence of subsequent conversion to other methods was higher in the LCX. The overall procedural success was $>90 \%$, and the difference of procedural success rate according to the target vessels was smaller than that of the device success rate. There were four complications: three coronary dissections in the RCA and one longitudinal compression of the proximal stent in the LCX.

At a median of 363 (IQR: 222-382 days), target-vessel revascularization was undertaken in 21 lesions (Kaplan-Meier estimates $5.5 \%$ at 1 year; $13.2 \%$ at 2 year), located at the treated site in 14 , distal to the treated site in five, and proximal in two. Only 1 patient experienced myocardial infarction (MI), who presented with stent thrombosis.

\section{Discussion}

The main findings of the present study are: 1) calcified lesions were more frequent in the RCA and LAD; proximal tortuosity was more frequent in the LCX and 2) the most common primary indication for the RCA and LAD was increasing back-up, whereas that for the LCX was improving alignment.

This study focused on the mechanistic effect of GEC as per target vessels. As shown in Figures $1 \mathrm{~B}-\mathrm{E}$, the supposed mechanistic effect of GEC to improve alignment between the catheter tip and the target lesion is different for each target vessel. For those lesions without bifurcation (Figures $1 \mathrm{~B}, \mathrm{C}$ ), the main effect of GEC is to decreases the $\Delta \theta$ by the stretch action and to decrease friction due to the smooth inner wall, as well as to increase back-up support by deep intubation [9]. As shown in Figure $1 \mathrm{D}$, the mechanics in LCX are different because of the non-existence of a supporting point due to the large side branch (LAD). GEC makes the supporting point, which results in a change in the model like Figure $1 \mathrm{E}$. This resembles the model of a shepherd's crook RCA with GEC. As discussed above, the supposed mechanics of improving alignment using GEC in each target vessel are different. More details are discussed in the Appendix and Supplementary Figures S1 and S2.

This study offers an important point for interventional cardiologists because the necessity of GEC differs with each target vessel's anatomy. With the RCA, some other techniques, such as using larger size guiding, buddy wire technique, stiffer guidewire, and anchoring balloon technique, can be helpful. However, with the acutely angled LCX, the operator must use GEC as the first choice. Moreover, in cases of severe calcified lesions, the smooth delivery of a drug-eluting stent and drug-coated balloon using GEC might improve the long-term prognosis [8]. In this regard, it is worthwhile to consider the differences in the primary indication for GEC according to the target vessels.

Only one study has previously described the primary indication for GEC between the RCA and left coronary artery (LCA) [6]. It was stated that the most common primary indication for GEC is to improve the alignment or selective contrast injection for the LCA and to increase back-up for the RCA. Nevertheless, as the bifurcation angle of the left main (LM)-LCX is larger than that of the LM-LAD [10], the primary indication for the LAD and LCX should be distinguished. If we integrate the primary indication for the LAD and LCX together and convert the ratio of the LAD and LCX (17 were used in LAD and 20 in LCX in reference [6]), the leading indication for the LCA is improving alignment.

In this study, the adverse event rates were acceptable, considering the lesion complexity and patients' highrisk background (increased age, high prevalence of CKD, multivessel disease, and chronic total occlusion). It is of great concern that $\mathrm{MI}$ for atheroma progression of the proximal coronary artery might be triggered by GEC deep intubation. In this regard, the result of the present study revealed the safety of GEC facilitated $\mathrm{PCI}$.

Although we adopted only one primary indication for each lesion, the actual mechanics were more complex. Furthermore, we ignored the deflection moment of catheters to simplify the model. However, considering the mechanistic effect of GEC, even in the simplified model, is important in choosing the best technique for each lesion.

\section{Conclusions}

The primary indication for GEC differed considerably among target vessels. Interventional cardiologists should consider the mechanistic effect of GEC to maximize its performance.

\section{Conflict of interest}

H.I. received lecture fees from Astellas Pharma Inc., Bayer Pharmaceutical Co., Ltd., Daiichi-Sankyo Pharma Inc., and MSD. T.M. received lecture fees from Bayer Yakuhin Ltd., Daiichi-Sankyo Co., Ltd., MSD K. K., Mitsubi- 
Table I. Angiographic and procedural characteristics according to the target vessels

\begin{tabular}{|c|c|c|c|c|}
\hline Lesion characteristics & $\begin{array}{l}\text { Right coronary artery } \\
n=124 \text { lesions }\end{array}$ & $\begin{array}{l}\text { Left anterior descending } \\
\text { artery } \\
n=80 \text { lesions }\end{array}$ & $\begin{array}{l}\text { Left circumflex artery } \\
n=47 \text { lesions }\end{array}$ & $P$-value \\
\hline Clinical indication for $\mathrm{PCl}$ : & & & & 0.10 \\
\hline Stable angina/silent ischemia & $88(71.0 \%)$ & $61(76.3 \%)$ & $35(74.5 \%)$ & \\
\hline NSTE-ACS & $15(12.1 \%)$ & $11(13.8 \%)$ & $10(21.3 \%)$ & \\
\hline STEMI & $21(16.9 \%)$ & $8(10.0 \%)$ & $2(4.3 \%)$ & \\
\hline \multicolumn{5}{|l|}{ Lesion complexity: } \\
\hline Type B2 or C lesion $^{\dagger}$ & $100(80.7 \%)$ & $76(95.0 \%)$ & $41(87.2 \%)$ & 0.01 \\
\hline Severe calcification & $77(62.1 \%)$ & $55(68.8 \%)$ & $16(34.0 \%)$ & 0.0004 \\
\hline Tortuosity & $47(37.9 \%)$ & $19(23.8 \%)$ & $43(91.5 \%)$ & $<0.0001$ \\
\hline Chronic total occlusion & $31(25.0 \%)$ & $13(16.3 \%)$ & $11(23.4 \%)$ & 0.31 \\
\hline Distal location & $53(42.7 \%)$ & $36(45.0 \%)$ & $36(57.5 \%)$ & 0.22 \\
\hline \multicolumn{5}{|l|}{ Procedure: } \\
\hline Radial access & $62(50.0 \%)$ & $49(61.3 \%)$ & $26(55.3 \%)$ & 0.20 \\
\hline Multivessel procedure & $9(7.3 \%)$ & $12(15.0 \%)$ & $14(29.8 \%)$ & 0.01 \\
\hline Radiation time [min] & $40[28,60]$ & $45[28,61]$ & $39[29,58]$ & 0.92 \\
\hline Contrast volume [min] & $85[55,120]$ & $95[65,150]$ & $96[61,148]$ & 0.37 \\
\hline Rotablator & $24(19.4 \%)$ & $27(33.8 \%)$ & $4(8.5 \%)$ & 0.002 \\
\hline Type of stent: & & & & 0.62 \\
\hline DES & $108(87.1 \%)$ & $73(91.3 \%)$ & $40(85.1 \%)$ & \\
\hline$\overline{D C B}$ & $9(7.3 \%)$ & $4(5.0 \%)$ & $3(6.4 \%)$ & \\
\hline POBA & $7(5.7 \%)$ & $3(3.8 \%)$ & $3(6.4 \%)$ & \\
\hline Total length of stent [mm] & $43.5[24.5,64]$ & $38[28,52]$ & $28[18,51]$ & 0.04 \\
\hline Minimum stent size [mm] & $3[2.5,3.5]$ & $2.5[2.25,3]$ & $2.5[2.25,2.9]$ & $<0.0001$ \\
\hline Number of stents used & $2[1,2]$ & $1[1,2]$ & $1[1,2]$ & 0.03 \\
\hline Guiding catheter size: & & & & 0.75 \\
\hline $6 \mathrm{Fr}$ & $51(41.1 \%)$ & $36(45.0 \%)$ & $21(44.7 \%)$ & \\
\hline $7 \mathrm{Fr}$ & $68(54.8 \%)$ & $43(53.8 \%)$ & $24(51.1 \%)$ & \\
\hline $8 \mathrm{Fr}$ & $5(4.0 \%)$ & $1(1.3 \%)$ & $2(4.3 \%)$ & \\
\hline Guiding catheter type: & & & & 0.06 \\
\hline Judkins type & $22(17.7 \%)$ & $24(30.0 \%)$ & $7(14.9 \%)$ & \\
\hline Backup type & $102(82.3 \%)$ & $56(70.0 \%)$ & $40(85.1 \%)$ & \\
\hline Guide extension size (6 Fr) & $114(91.4 \%)$ & $74(92.5 \%)$ & $44(93.6 \%)$ & 0.93 \\
\hline Situation for guide extension: & & & & 0.51 \\
\hline Balloon delivery & $56(45.2 \%)$ & $28(35.0 \%)$ & 19 (40.4\%) & \\
\hline Stent delivery & $43(34.7 \%)$ & $34(42.5 \%)$ & 19 (40.4\%) & \\
\hline Intravascular ultrasound delivery & $16(12.9 \%)$ & $6(7.5 \%)$ & $6(12.8 \%)$ & \\
\hline Selective contrast injection & $3(2.4 \%)$ & $5(6.3 \%)$ & $1(2.1 \%)$ & \\
\hline Other & $6(4.8 \%)$ & $7(8.8 \%)$ & $2(4.3 \%)$ & \\
\hline Complication & $3(2.4 \%)$ & $0(0.0 \%)$ & $1(2.1 \%)$ & 0.21 \\
\hline Device success & $119(96.0 \%)$ & $78(97.5 \%)$ & $42(89.4 \%)$ & 0.14 \\
\hline Procedure success & $120(96.8 \%)$ & 77 (96.3\%) & $43(91.5 \%)$ & 0.36 \\
\hline Switch to other method & $8(6.5 \%)$ & $6(7.5 \%)$ & $7(14.9 \%)$ & 0.24 \\
\hline
\end{tabular}

Values are the mean + standard deviation (SD) $n(\%)$, or median (interquartile range) as appropriate. Continuous variables were compared using ANOVA, and categorical variables were compared using the $\chi^{2}$ test. Type B2 or C lesion according to the American College of Cardiology/American Heart Association classification. PCI - percutaneous coronary intervention; NSTE-ACS - non-ST elevation acute coronary syndrome, STEMI - ST elevation myocardial infarction, DES - drug-eluting stent, BMS - bare-metal stent. 
A

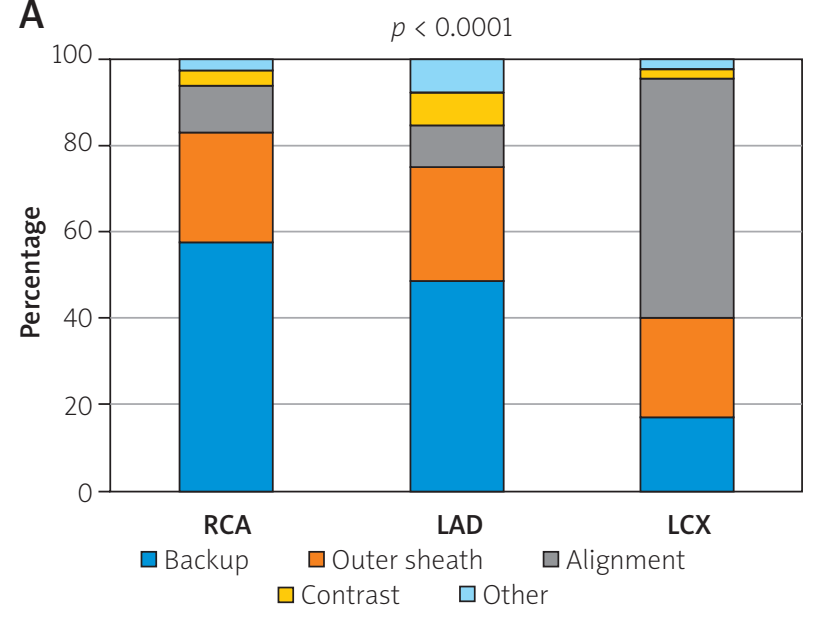

C

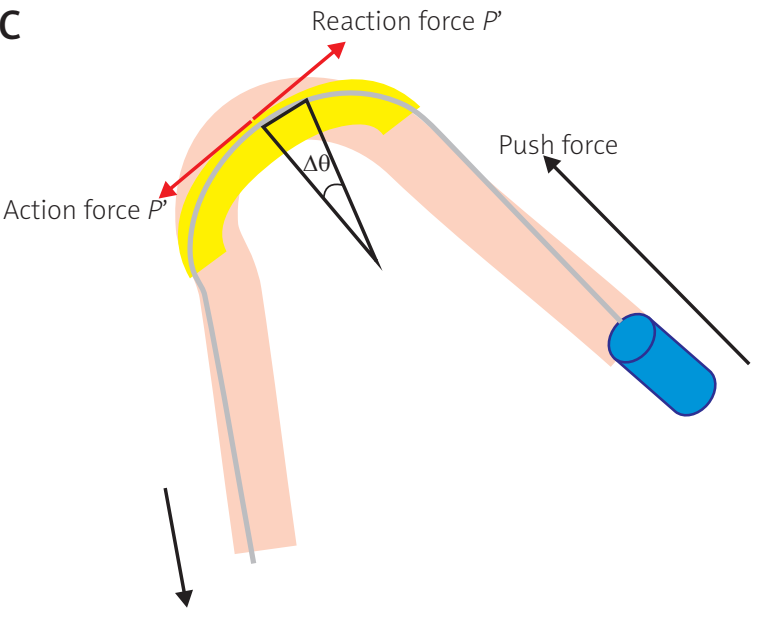

Tip force

$E$

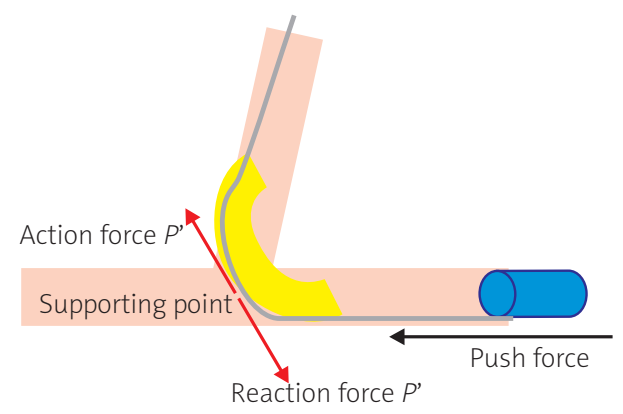

shi Tanabe Pharma Co., Nippon Boehringer Ingelheim Co., Ltd. Department of Cardiology, Nagoya University Graduate School of Medicine received research grants from Astellas Pharma Inc., Daiichi-Sankyo Co., Ltd., Dainippon Sumitomo Pharma Co., Ltd., Kowa Co., Ltd., MSD K. K., Mitsubishi Tanabe Pharma Co., Nippon Boehringer Ingelheim Co., Ltd., Pfizer Japan Inc., and Teijin Pharma Ltd.

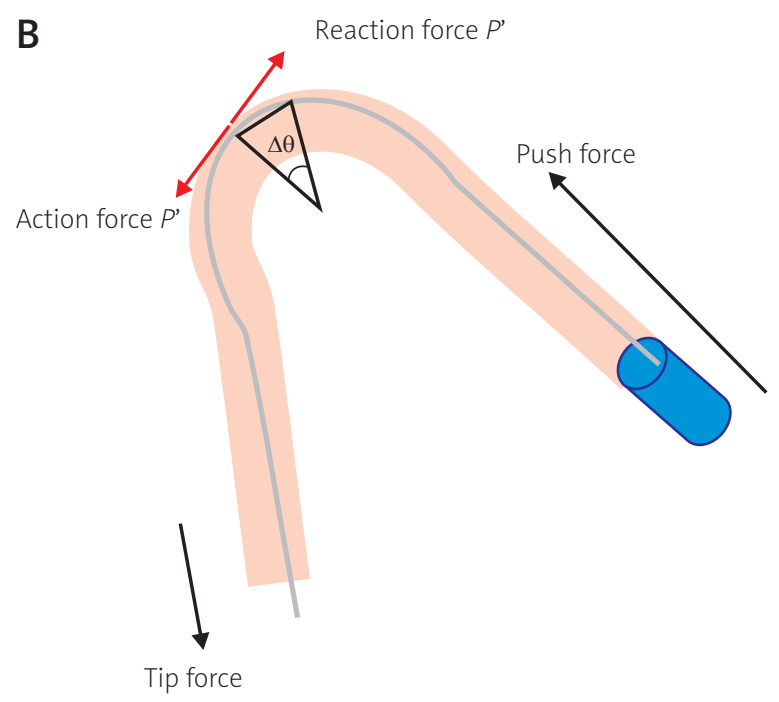

D

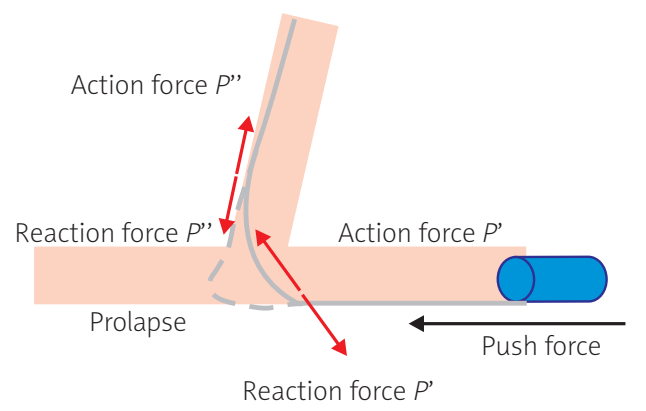

Figure 1. Primary indication for guide extension catheter (GEC) according to the three target vessels and mechanistic effect of GEC in the right coronary artery (RCA) and left circumflex (LCX). $A$ - the primary indication for GEC according to the target vessels. B - the shepherd's crook RCA model. The push force is captured at the vertex of the angle. C - the effect of GEC in the shepherd's crook RCA model. D - the acutely angled LCX model. The force escaping into the left anterior descending artery and the catheter shaft might prolapse. E - the effect of GEC for the acutely angled LCX. GEC can provide the supporting point and the push force is effectively transmitted

\section{References}

1. Duong T, Christopoulos G, Luna M, et al. Frequency, indications, and outcomes of guide catheterextension use in percutaneous coronary intervention. J Invasive Cardiol 2015; 27: E211-5.

2. Hirokami M, Saito S, Muto H. Anchoring technique to improve guiding catheter support in coronary angioplasty of chronic total occlusions. Catheter Cardiovasc Interv 2006; 67: 366-71.

3. Burzotta F, Trani C, Mazzari MA, et al. Use of a second buddy wire during percutaneous coronary interventions: a simple solu- 
tion for some challenging situations. I Invasive Cardiol 2005; 17: 171-4.

4. Zhang Q, Zhang RY, Kirtane AJ, et al. The utility of a 5-in-6 double catheter technique in treating complex coronary lesions via transradial approach: the DOCA-TRI study. Eurolntervention 2012; 8: 848-54.

5. Alhaddad IA. Novel double catheter technique in complex percutaneous coronary interventions. Catheter Cardiovasc Interv 2006; 67: 912-4.

6. de Man FH, Tandjung K, Hartmann M, et al. Usefulness and safety of the GuideLiner catheter to enhance intubation and support of guide catheters: insights from the Twente GuideLiner registry. Eurolntervention 2012; 8: 336-44.

7. Sharma D, Shah A, Osten M, et al. Efficacy and safety of the GuideLiner mother-in-child guide catheter extension in percutaneous coronary intervention. J Interv Cardiol 2017; 30: 46-55.

8. Yoshida R, Ishii H, Morishima I, et al. Impact of adjunctive use of guide extension catheter on midterm outcome of drug-coated balloon angioplasty. Eurolntervention 2019; 15: 688-91.

9. Takeshita S, Takagi A, Saito S. Backup support of the mother-child technique: technical considerations for the size of the mother guiding catheter. Catheter Cardiovasc Interv 2012; 80: 292-7.

10. Kawasaki T, Koga H, Serikawa T, et al. The bifurcation study using 64 multislice computed tomography. Catheter Cardiovasc Interv 2009; 73: 653-8. 


\section{Appendix}

The supposed mechanics of the RCA are shown in Supplementary Figure S1. Supplementary Figure S1 A reveals the relationship of force and angle $\Delta \theta$ in the angulated point. When one point of the catheter shaft is advanced by power $(P)$, there generates a perpendicular force $(N)$ from the vessel wall, a frictional force $(S)$, and a reactive force $\left(P^{\prime}\right)$ from the next point. $P^{\prime}$ is equal to the advancing power $\left(-P^{\prime}\right)$. The following formulas in the tangent $(\vec{t})$ and perpendicular $(\vec{n})$ directions arise:

$$
\begin{gathered}
\vec{t}: P \cos (\Delta \theta / 2)-S=P^{\prime} \cos (\Delta \theta / 2) \\
\vec{n}: P \sin (\Delta \theta / 2)+P^{\prime} \sin (\Delta \theta / 2)=N \\
S=\mu^{\prime} N \\
0 \leq \Delta \theta<\pi
\end{gathered}
$$

Thus, the advancing power $\left(-P^{\prime}\right)$ is derived according to the following equation:

$$
P^{\prime}=\left[1-\mu^{\prime} \tan (\Delta \theta / 2)\right] P /\left[1+\mu^{\prime} \tan (\Delta \theta / 2)\right]
$$

The graph of $P^{\prime}$ is shown in Supplementary Figure S1 B (dashed line, $\mu^{\prime}=0.01$; fine solid line, $\mu^{\prime}=0.1$; heavy solid line, $\mu^{\prime}=0.3$ ). The more the angle $\Delta \theta$ increases, the less $P^{\prime}$ decreases. Also, at the same angle $\Delta \theta$, the more the friction increase, the less $P^{\prime}$ decreases.

The angle $\Delta \theta$ is larger in the acutely angled RCA (Supplementary Figure S1 D) than in the normal smooth angle model (Supplementary Figure S1 C), which resulted in decreased P' (Supplementary Figure S1 B). With GEC, the $\Delta \theta$ decreases by the stretch action of GEC, and the $\mu^{\prime}$ decreases because of the smoothness of the GEC inner wall (Supplementary Figure S1 E).

The supposed physics of the LCX model are shown in Supplementary Figure S2. There is no supporting point on the corner due to the large side branch (LAD); therefore the mechanics are different. When point 1 (left main distal) is advanced by power $(P)$, there generates perpendicular force $(N)$ from the vessel wall, frictional force $(S)$, and reactive force $\left(P^{\prime}\right)$. At the point 2 (the carina point), there generates perpendicular force $\left(N^{\prime}\right)$ from the vessel wall, frictional force $\left(S^{\prime}\right)$, and reactive force $\left(P^{\prime \prime}\right)$. The following formulas arise:

$$
\begin{aligned}
& \left\{\begin{array}{c}
\vec{t}: P-S=P^{\prime} \cos \theta \\
\vec{n}: P^{\prime} \sin \theta=N
\end{array}\right. \\
& \left\{\begin{array}{c}
\vec{t}^{\prime}: P^{\wedge}{ }^{\prime \prime}+S=P^{\prime} \sin (\theta-\varphi) \\
\vec{n}^{\prime} \cdot P^{\prime} \cos (\theta-\varphi)=N^{\prime}
\end{array}\right. \\
& S=\mu^{\prime} N \\
& S^{\prime}=\mu^{\prime} N^{\prime} \\
& 0 \leq \theta<\pi / 2 \\
& 0 \leq \varphi<\pi / 2
\end{aligned}
$$

Thus, the advancing power (- $\left.P^{\prime \prime}\right)$ is derived according to the following equation:

$$
P^{\prime \prime}=\left[\sin (\theta-\varphi)-\mu^{\prime} \cos (\theta-\varphi)\right] P /\left[\cos \theta+\mu^{\prime} \sin \theta\right]
$$

In the case of $\varphi=0$ (the angle between LMT-LCX is $90^{\circ}$ ), the equation is as follows:

$$
P^{\prime \prime}=\left[\tan \theta-\mu^{\prime}\right] P /\left[1+\mu^{\prime} \tan \theta\right]
$$

The graph of this equation ( $P$ ") is shown in Supplementary Figure S2 B (dashed line, $\mu^{\prime}=0.01$; fine solid line, $\mu^{\prime}=0.1$; heavy solid line, $\mu^{\prime}=0.3$ ).

In the case of $\theta=\pi / 2$, the equation is as follows:

$$
P^{\prime \prime}=\left[\left(1-\mu^{\prime}\right) \cos \phi-\left(1+\mu^{\prime}\right) \sin \phi\right] P /\left[1+\mu^{\prime}\right]
$$

The graph of this equation ( $\left.P^{\prime \prime}\right)$ is shown in Figure S2 C (dashed line, $\mu^{\prime}=0.01$; fine solid line, $\mu^{\prime}=0.1$; heavy solid line, $\left.\mu^{\prime}=0.3\right)$. In this situation, the more the angle $\theta$ increases, the more $P$ " increases; the more the angle $\phi$ increases, the less $P$ "' decreases. At the same angle $\theta$ and $\phi$, the more the friction increases, the less $P^{\prime}$ decreases. Furthermore, the force sometimes escaping into the LAD and the catheter shaft prolapses (Supplementary Figure S2 D). In this situation, applying GEC is very useful. Because the GEC forms the supporting point, the model changes to that shown in Supplementary Figure S2 E, which is the same situation as Supplementary Figure S1 E. Comparing the graph of Supplementary Figure S1 B with Supplementary Figure S2 C, the power in the situation of Supplementary Figure S1 B is much larger than that in Supplementary Figure S2 C. 